\title{
Icotinib hydrochloride enhances the effect of radiotherapy by affecting DNA repair in colorectal cancer cells
}

\author{
HONG MA, JIANPING BI, TAO LIU, YANG KE, SHENG ZHANG and TAO ZHANG \\ Cancer Center of Wuhan Union Hospital, TongJi Medical College, Huazhong University \\ of Science and Technology, Wuhan, Hubei 430022, P.R. China
}

Received September 11, 2014; Accepted November 27, 2014

DOI: $10.3892 /$ or.2014.3699

\begin{abstract}
The aim of the present study was to explore the efficacy and mechanism of the radiosensitisation of icotinib hydrochloride (IH), a novel oral epidermal growth factor receptor-tyrosine kinase activity inhibitor, by evaluating the changes in tumour cell double-strand breaks (DSBs) repair, cell cycle and apoptosis following a combination of $\mathrm{IH}$ and radiotherapy (RT) in human colorectal adenocarcinoma cell lines. The HT29 and HCT116 human CRC cell lines were treated with IH and/or radiation. Effects on cell viability and cell cycle progression were measured by MTT, a clonogenic survival assay, and flow cytometry. Immunofluorescent staining and western blot analysis were applied to detect the expression of $\gamma-\mathrm{H} 2 \mathrm{AX}$ and 53BP1 in the different treatment groups. Finally, the in vivo effect on the growth of CRC xenografts was assessed in athymic nude mice. IH inhibited the proliferation and enhanced the radiosensitivity in HT29 and HCT116 CRC cells lines. IH combined with radiation increased cell cycle arrest in the $\mathrm{G} 2 / \mathrm{M}$ phase compared to the other treatments in the HT29 cell line $(\mathrm{P}<0.05)$. Similarly, cell cycle arrest occurred in the HCT116 cell line, although this increase did not result in significant differences in the RT group $(\mathrm{P}>0.05)$. IH combined with radiation significantly inhibited the expression of $\gamma$-H2AX and 53BP1 based on results of immunofluorescent staining and western blot analysis. In vivo, IH plus radiation significantly inhibited the tumour growth compared to either agent independently. In conclusion, IH significantly increased the radiosensitivity of HT29 and HCT116 cells in vitro and in vivo. Radiation combined with EGFR blockade inhibited
\end{abstract}

Correspondence to: Dr Hong Ma or Dr Tao Zhang, Cancer Center of Wuhan Union Hospital, TongJi Medical College, Huazhong University of Science and Technology, 1277 Jiefang Road, Wuhan, Hubei 430022, P.R. China

E-mail: wudajianzhu2004@163.com

E-mail: zhangtao_xiaohua@126.com

Key words: colorectal cancer, epidermal growth factor receptor, icotinib hydrochloride, radiosensitivity, double-strand breaks repair tumour proliferation, increased apoptosis, prolonged G2/M arrest and significantly enhanced DNA injury in colorectal cancer. These data support the clinical trials of biologically targeted and conventional therapies in the treatment of cancer.

\section{Introduction}

Colorectal cancer is the third most prevalent cancer worldwide, and its incidence rate in Asia increases each year. Surgery is the primary means to effectively treat colorectal cancer, however, the relapse rate can be as high as $30 \%$ for partially terminal colorectal cancer patients even after radical surgery and multidisciplinary treatment. Of these patients, only $7-20 \%$ of recurrences can be treated with surgery again (1-3). Radiation therapy is another important type of cancer treatment. Results of previous studies showed that concurrent radiotherapy and chemotherapy prior to surgery can downstage advanced colorectal cancer, improve the surgical resection rate, reduce the local recurrence rate and improve progression-free survival benefits $(4,5)$. In current clinical practice, radiosensitisers for colorectal cancer mainly include fluoropyrimidine-based medicines. However, their treatment toxicity is a major factor that limits the application of concurrent radiotherapy and chemotherapy (6).

The recent emergence of targeting drugs provides a more safe and effective way to achieve radiosensitisation. Epidermal growth factor receptor (EGFR), which belongs to the ErbB receptor tyrosine kinase family, is an important growth factor. EGFR binding to its ligand phosphorylates the intracellular tyrosine kinase receptor through conformational changes, activates a number of signalling pathways and thus plays an important role in tumour proliferation, differentiation, metastasis, and angiogenesis (7,8). Approximately 60-80\% of colorectal cancer tumour tissue expresses EGFR, and its expression is closely associated with cancer progression, poor prognosis, treatment resistance and especially radiation resistance $(9,10)$. Thus, the EGFR signalling pathway is an important target for cancer treatment, and it has been shown that EGFR inhibitors combined with radiotherapy can enhance the efficacy of radiotherapy, rendering it an effective radiosensitising drug $(11,12)$.

Two types of drugs have been designed for selective blockade of EGFR signalling,including monoclonal antibodies (mAbs) specific for EGFR, such as cetuximab and 
nimotuzumab, and small molecule inhibitors of the tyrosine kinase activity of EGFR (EGFR-TKI), such as gefitinib and erlotinib. Although the former type of drug exerted a radiosensitising effect in some colon cancer cell lines, the effect is subject to the expression of certain genes, such as $K-R A S$ and $B R A F$, which increases treatment uncertainty $(13,14)$. In 2002 , Williams et al found that the small molecule TKI gefitinib can promote the reaction of colon cancer cell lines ( $\mathrm{LoVO}$ ) to radiation in vitro (15). Subsequent studies of a mouse in vivo tumour model found that gefitinib in combination with radiation therapy significantly inhibited tumour proliferation compared with radiotherapy alone, which confirms the radiosensitisation of gefitinib in colorectal cancer (15). However, in studies of other TKI inhibitors, such as the radiosensitisation of colorectal cancer by erlotinib and (ZEGFR $\left.{ }_{19072}\right)$, the sensitising effects of erlotinib and (ZEGFR $\left.{ }_{19072}\right)$ were not equivalent to that of gefitinib (16). Icotinib hydrochloride ( $\mathrm{IH}$ ) is a new oral epidermal growth factor receptor tyrosine kinase inhibitor, a quinazolinetype drug, with targets and mechanisms similar to those of gefitinib $(17,18)$. It is a reversible EGFR intracellular TKI with an efficacy equal to that of gefitinib both in vitro and preclinical studies (18). This drug has already shown a significant inhibitory effect on colon cancer at the cell level (17). However, its superiority or equivalence to the effect of gefitinib when combined with radiation therapy remains unclear.

Owing to the lethal damage induced by radiation therapy-DNA double-strand breaks (DSBs), interfering with DSBs repair has become an important radiosensitisation strategy $(19,20)$. EGFR-TKI has been found to affect the key components of intracellular DNA repair to sensitise cells to radiotherapy (21). Therefore, this present study was performed to evaluate the radiosensitisation efficacy of a combination of IH and radiotherapy in human colorectal cancer cell lines via in vitro and in vivo models and to investigate whether the effects of the radiosensitisation are correlated with changes in tumour cell apoptosis, cell cycle and DNA repair, by examining the expression of the phosphorylated histone $\mathrm{H} 2 \mathrm{AX}(\gamma-\mathrm{H} 2 \mathrm{AX})$ and 53BP1 (P53 binding protein 1) proteins during DSB repair.

\section{Materials and methods}

Cell lines and cultures. HT29 and HCT116 cells were obtained from the laboratory of the General Surgical Department, Union Hospital, Tongji Medical College, Huazhong University of Science and Technology (Hubei, China) and maintained in RPMI-1640 medium supplemented with $10 \%$ foetal bovine serum. The cells were cultured in a humidified incubator with $5 \% \mathrm{CO}_{2}$ at $37^{\circ} \mathrm{C}$. IH was provided by Zhejiang Beta Pharma Ltd. (Hangzhou, China), and dissolved in 100\% dimethyl sulfoxide (DMSO) (Sigma, St. Louis, MO, USA) to a final concentration of $30 \mathrm{mg} / \mathrm{ml}$ and stored at $-20^{\circ} \mathrm{C}$.

MTT assay. A total of $0.5-1 \times 10^{5}$ exponentially growing cells were seeded in 96-well micro-titre plates (Corning Inc., New York, NY, USA) and were treated with different concentrations of IH $(0.01,0.03,0.1,0.3$ and $0.9 \mathrm{mg} / \mathrm{ml})$ following incubation in growth medium overnight at $37^{\circ} \mathrm{C}$. After $24 \mathrm{~h}$ of $\mathrm{IH}$ addition, a microculture tetrazolium (MTT) assay was performed by adding $20 \mu \mathrm{l}$ of 3-(4,5-diethyl-2-thiazolyl)-2,5-diphenyltetrazolium bromide $(5 \mathrm{mg} / \mathrm{ml})$ (Sigma) to each well for $4 \mathrm{~h}$ at $37^{\circ} \mathrm{C}$ to allow metabolically active cells to generate formazan crystals from MTT. The medium was aspirated and $150 \mu \mathrm{l}$ of DMSO (Sigma) was added to dissolve the formazan. After $10 \mathrm{~min}$, the above mixture was measured in a microplate reader (Bio-Tek, Winooski, VT, USA) at a wavelength of $490 \mathrm{~nm}$. The percentage inhibition rate was calculated as: (1 - OD value of experimental group/control group OD) $\mathrm{x} 100 \%$. The $\mathrm{IC}_{20}$ was selected as the subsequent experiment concentration.

Clonogenic assays.HCT116 and HT29 cells were collected from exponential phase cultures by trypsinisation, counted, and then seeded in 6-well plates (Corning Inc.) with densities varying from $1 \times 10^{2}$ to $5 \times 10^{3}$ cells/well depending on the intended radiation dose. According to the MTT assay, the $\mathrm{IC}_{20}$ was selected for subsequent experiments. IH (0.03 and $0.06 \mathrm{mg} / \mathrm{ml}$ for HCT116 and HT29 cells, respectively) was added $24 \mathrm{~h}$ prior to radiation exposure with single doses ranging from 0 to $10 \mathrm{~Gy}$. Irradiation treatments in this study were performed on a clinically calibrated Siemens Oncor accelerator using $6 \mathrm{MV}$ photons at a nominal dose rate of $3 \mathrm{~Gy} / \mathrm{min}$ (Siemens Medical Solutions, Concord, CA, USA). After $24 \mathrm{~h}$, the medium containing IH or DMSO was substituted with medium that only contained $10 \%$ foetal bovine serum. After 10-14 days, the cells were fixed with methanol (Guge, Wuhan, China) and stained with $10 \%$ crystal violet (Guge). The colonies were counted, and a grouping of $>50$ cells was considered a colony. The plating efficiency (PE) was the percentage of cells seeded that grew into colonies under special culture conditions. The survival fraction, expressed as a function of the radiation dose, was calculated as: Survival fraction $=$ colonies counted/(cells seeded $\mathrm{x}$ PE/100). Experiments were repeated three times.

Assessment of cell apoptosis. After $24 \mathrm{~h}$ of seeding, the cells were exposed to IH ( 0.06 and $0.03 \mathrm{mg} / \mathrm{ml}$ for HT29 and HCT116 cells) for $24 \mathrm{~h}$, radiotherapy (10 Gy) for $24 \mathrm{~h}$, or the combination treatment. The trypsinised cells were re-suspended in $1 \mathrm{X}$ binding buffer at a concentration of $2 \times 10^{5}$ cells $/ \mathrm{ml}$, and Annexin V-FITC and propidium iodide (PI) (Sigma) were added. The cells were incubated for an additional $15 \mathrm{~min}$ at room temperature in the dark and then subjected to analysis with flow cytometry (BD Biosciences, San Jose, CA, USA). A minimum of 10,000 cells in each sample were analysed, and the data were analysed using the Cell Quest software (BD Biosciences).

Cell cycle analysis. Cells were collected after $24 \mathrm{~h}$ of exposure to $\mathrm{IH}, 10 \mathrm{~Gy}$ radiation, or the combination treatment. The cells were washed with PBS (Boster, Wuhan, China) and harvested by trypsinisation. After centrifugation at ? for ?, the cell pellets were fixed in $70 \%$ cold ethanol. Following removal of the ethanol by centrifugation, the cells were stained with a DNA staining solution $(20 \mu \mathrm{g} / \mathrm{ml}$ of propidium iodide and $10 \mu \mathrm{g} / \mathrm{ml}$ of RNase A) for $30 \mathrm{~min}$. The stained cells were then suspended and immediately subjected to analysis using a flow cytometer (BD Biosciences). The resulting DNA distribution was analysed by ModFit for the proportion of cells in the sub-G0, G1, S, and G2-M phases of the cell cycle. A minimum of 10,000 cells was counted in each sample, and the cell cycle distribution was calculated using the Cell Quest software (BD Biosciences). 
Immunofluorescent staining for $\gamma-H 2 A X$ and 53BPl. Cells $\left(2 \times 10^{5}\right)$ were plated in chamber slides. IH was added following cell adhesion, resulting in a final concentration of 0.06 and $0.03 \mathrm{mg} / \mathrm{ml}$ for HT29 and HCT116 cells, respectively. After $24 \mathrm{~h}$, the cells were treated with radiation. After another $24 \mathrm{~h}$, the medium was aspirated and the cells were fixed with $4 \%$ paraformaldehyde. The cells were rinsed with PBS three times and permeabilised with $0.2 \%$ Triton-X-100 (Boster) for $20 \mathrm{~min}$ at $4^{\circ} \mathrm{C}$. The cells were rinsed with PBS three times again and blocked with 5\% BSA for $1 \mathrm{~h}$ at room temperature. The cells were again washed with PBS three times and anti$\gamma$-H2AX antibody (1:200, Abcam, Cambridge, MA, USA) and anti-53BP1 antibody (1:200, Bethyl, Inc., Montgomery, TX, USA) were added at a dilution of 1:800 and 1:200 in 1\% BSA, respectively. Subsequently, the slides were incubated overnight at $4^{\circ} \mathrm{C}$. The cells were rinsed with PBS prior to incubation with cyanine 3 (CY3)-labelled secondary antibody (Protientech Group, Chicago, IL, USA) at a dilution of 1:200 in 1\% BSA for $1 \mathrm{~h}$ in the dark. The secondary antibody was aspirated, and the cells were rinsed with PBS three times and incubated with 4',6-diamidino-2-phenylindole dihydrochloride (DAPI) (Vector Laboratories Inc., Burlingame, CA, USA) in the dark for $10 \mathrm{~min}$. The slides were examined using a fluorescent microscope. The images were captured with a confocal laser scanning microscope (CLSM) (Olympus, Tokyo, Japan) equipped with a camera. For each sample, the $\gamma-\mathrm{H} 2 \mathrm{AX}$ and $53 \mathrm{BP} 1$ foci were determined in $\geq 100$ cells.

Western blot analyses. The cells were seeded and allowed to adhere in complete medium overnight. They were then treated with or without IH $(0.06$ and $0.03 \mathrm{mg} / \mathrm{ml}$ for HT29 and HCT116 cells) $24 \mathrm{~h}$ prior to irradiation with a $10 \mathrm{~Gy}$ dose, and the cells were lysed with a lysis buffer (Beyotime, Wuhan, China). The protein lysates were harvested and centrifuged, and the supernatants were collected. The proteins were separated via 10 or $12 \%$ sodium dodecyl sulphate (SDS)/polyacrylamide gel electrophoresis and then transferred to polyvinyl difluoride membranes (Millipore, Billerica, MA, USA). The membranes were then blocked with $5 \%$ bovine serum albumin or milk and finally incubated with $\gamma$-H2AX (Abcam), 53BP1 antibody (Bethyl, Inc.) or $\beta$-actin antibody (Santa Cruz Biotechnology, Inc., Santa Cruz, CA, USA). The membranes were incubated with the primary antibodies overnight at $4^{\circ} \mathrm{C}$ and rinsed with TBST three times, followed by incubation with secondary antibodies labelled with horseradish peroxidase (HRP) (Protientech Group). The bands were then visualised using enhanced chemiluminescence (ECL) (Pierce, Rockford, IL, USA).

Assay for tumour growth in athymic nude mouse model. Exponential phase HT29 and HCT116 cells were prepared at a concentration of $2 \times 10^{7}$ cells $/ \mathrm{ml}$ in serum-free RPMI medium. Female athymic nude mice (nu/nu, body weight, 20-25 g, 8-12 weeks of age) were obtained from Beijing HFK Bioscience Co., Ltd. The mice were provided with sterilised food and water and housed in a barrier facility with 12-h light/dark cycles in laminar flow hoods at a constant temperature and humidity for the entire course of the experiments and supplied with a standard laboratory diet and water. The tumour xenografts were established via the subcutaneous injection of a $0.2 \mathrm{ml}$ volume of the prepared cell stock into the right hind leg. After 7 days,

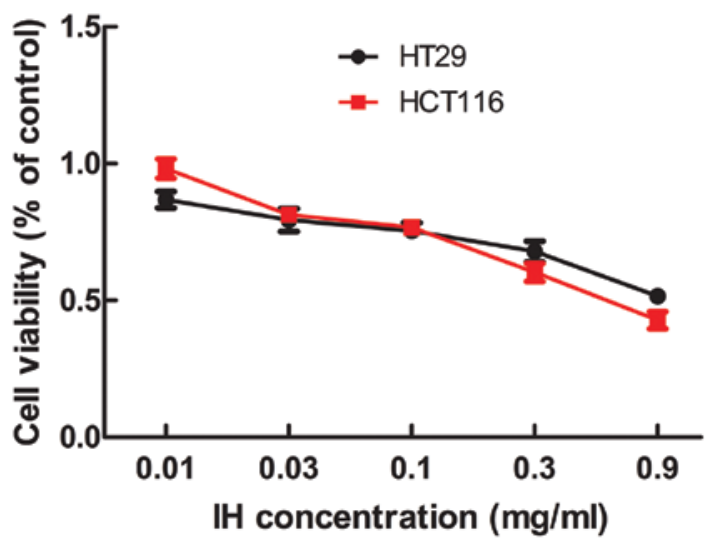

Figure 1. Viability of HT29 and HCT116 cells treated with icotinib at different ion concentrations, expressed as a percentage of the control cells. Error bars show the standard error of the mean (SEM) for three independent experiments.

when the diameter of each tumour increased to $10 \mathrm{~mm}$, the mice were pooled and randomly assigned to 4 groups (control, $\mathrm{IH}$ alone, radiation alone, and radiation in combination with $\mathrm{IH})$ of 6 animals each. IH ( $35 \mathrm{mg} / \mathrm{kg})$ was administered via oral gavage once a day for 5 days, and locoregional irradiation was administered in a single $2 \mathrm{~Gy}$ fraction once a day (6-MV linear accelerator, MDX, Siemens) for 5 days. The tumour size was measured using callipers every two days. Animal protocols and studies were conducted in accordance with the guidelines of the Institutional Animal Care and Use Committee of the Korea Institute of Radiological and Medical Sciences, Korea.

The tumour volumes (V) were determined according to the two axes of the tumour (L, longest axis; W, shortest axis). The volume was calculated according to the formula: Tumour volume $\left(\mathrm{mm}^{3}\right)=\left(\mathrm{L} \mathrm{x} \mathrm{W}^{2}\right) / 2 \mathrm{~mm}^{3}$, where $\mathrm{L}$ and $\mathrm{W}$ are the shortest and the longest diameter.

Statistical analysis. SPSS 13.0 software was used for the data analysis. The data are reported as the mean \pm SEM and analysed using one-way ANOVA to compare means in multiple groups. A q-test was used for the pairwise comparison among groups. A difference was regarded as significant if $\mathrm{P}<0.05$.

\section{Results}

Effect of icotinib on cell viability. The effect of icotinib on cell viability was measured with the MTT assay. The inhibitory effect of icotinib positively correlates with the drug concentration (Fig. 1). The $\mathrm{IC}_{50}$ (median inhibition concentration) and $\mathrm{IC}_{20}$ (inhibiting concentration 20) values were obtained. The $\mathrm{IC}_{20}$ value was selected as the drug concentration for subsequent experiments, with values of 0.06 and $0.03 \mathrm{mg} / \mathrm{ml}$ for HT29 and HCT116.

Influence of icotinib on the radiation sensitivity of human colorectal cancer cell lines. To determine the radiosensitising effect of icotinib on the HT29 and HCT116 colorectal cell lines, a clonogenic formation assay was performed. The result was compared to gefitinib combined with radiation, similar to icotinib. $\mathrm{IC}_{20}$ values of gefitinib on HT29 and HCT116 cells were explored and applied for further study of radiosensitisation. 

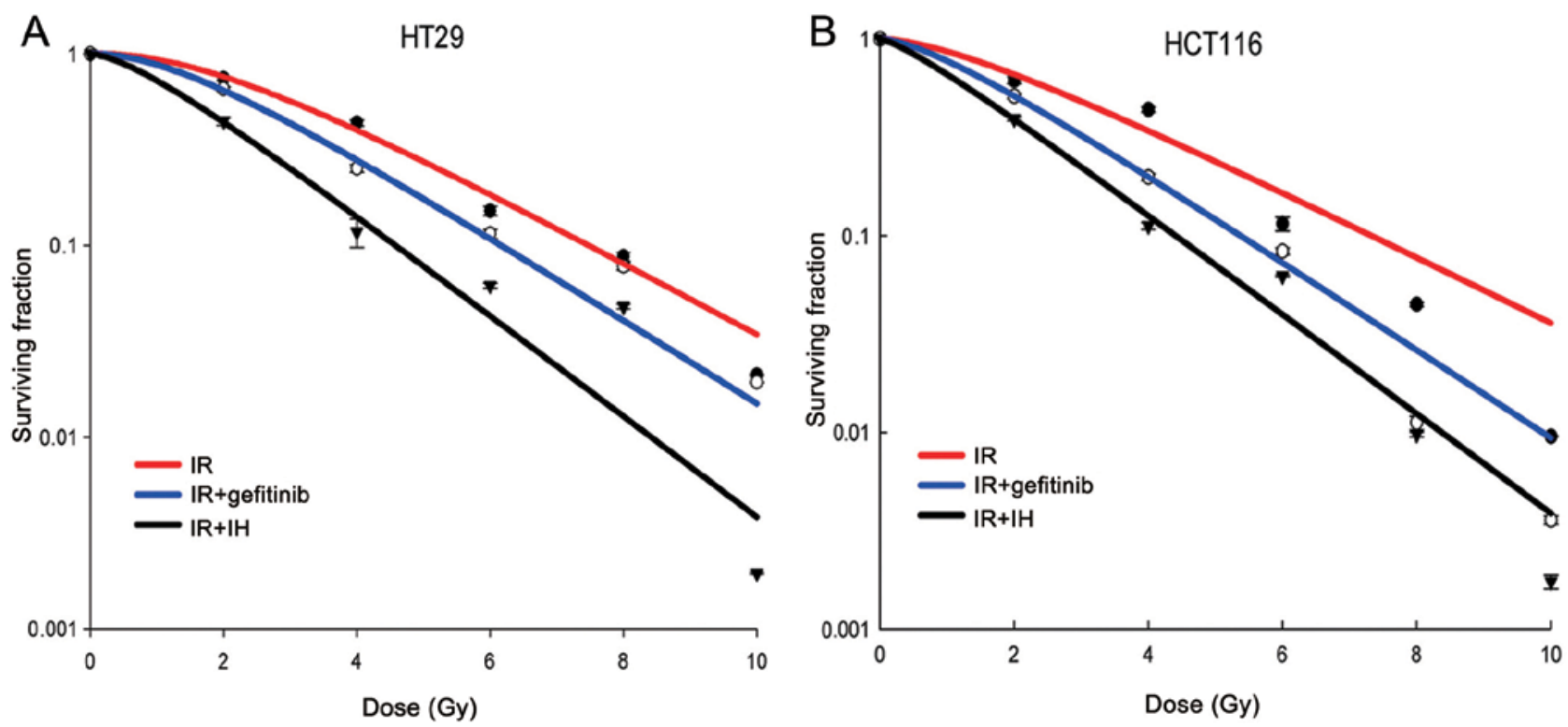

Figure 2. The effect of icotinib on radiosensitivity from the clonogenic assay. The cells were treated with irradiation only (red line) and gefitinib or icotinib $1 \mathrm{~h}$ prior to radiation (blue and dark line). The L-Q model was used to fit the experimental data. Error bars show the standard error of the mean (SEM) for $\mathrm{n}=3$ independent experiments. (A) Survival curve of HT29 and (B) HCT116 cells with different treatments.

Table I. The main parameters of cell survival curves of HT29 and HCT116 following irradiation.

\begin{tabular}{lllllllll}
\hline & \multicolumn{3}{c}{ HT29 } & & \multicolumn{3}{c}{ HCT116 } \\
\cline { 2 - 4 } \cline { 6 - 8 } Parameter & IR & Gefitinib+IR & IH+IR & & IR & Gefitinib+IR & IH+IR \\
\hline D0 & 2.31 & 2.00 & 1.65 & 2.58 & 1.94 & 1.71 \\
Dq & 1.80 & 1.51 & 0.99 & 1.51 & 1.15 & 0.81 \\
$\mathrm{~N}$ & 2.61 & 2.26 & 1.63 & 1.75 & 1.63 & 1.32 \\
SF2 & 0.76 & 0.64 & 0.44 & & 0.66 & 0.51 & 0.39 \\
SER & & 1.19 & 1.73 & & 1.29 & 1.69 \\
\hline
\end{tabular}

D0, mean lethal dose; Dq, quasi-threshold dose; N, extrapolation number; SF2, surviving fraction at $2 \mathrm{~Gy}$, IR, irradiation.

The L-Q model was used to investigate the difference in radiosensitivity from gefitinib or $\mathrm{IH}$ combined with irradiation or irradiation alone. The results showed that the Dq, Do and $\mathrm{N}$ values were reduced in response to treatment with gefitinib or icotinib combined with irradiation when compared with irradiaton alone for HT29 and HCT116. Furthermore, icotinib exhibited a stronger ability to reduce the Dq, Do and $\mathrm{N}$ values compared to gefitinib. Radiotherapy alone decreased the SF2 values of HT29 and HCT116 to 76 and $66 \%$, whereas when gefitinib was added, the SF2 values decreased to 64 and 51\%, respectively. Addition of icotinib resulted in the reduction of SF2 values to 44 and $39 \%$, respectively, suggesting that icotinib more effectively sensitises colorectal cancer cells to radiation as compared to gefitinib (Table I, Fig. 2).

Effect of icotinib in combination with radiotherapy on cell apoptosis. To determine whether icotinib combined with radiotherapy increased apoptosis, HT29 or HCT116 cells were treated with vehicle or IH with or without radiotherapy [control; IH alone; radiotherapy (RT) alone; IH+RT], and the cell apoptotic rate was then detected via flow cytometry $24 \mathrm{~h}$ after the treatment (Fig. 3). In HT29 cells, the apoptotic rate of the combined group was $26.97 \pm 7.15 \%$, while it was $14.17 \pm 5.44 \%$ in the monotherapy group, $15.81 \pm 3.31 \%$ in the RT group and $12.5 \pm 2.93 \%$ in the control group. Significant differences were observed between the combined group and the remaining three groups $(\mathrm{P}<0.01)$. For HCT116 cells in the control group, the apoptotic rate was $10.08 \pm 0.62 \%$, while it was $12.21 \pm 2.71 \%$ in the IH alone group, $18.45 \pm 3.45 \%$ in the RT group and $28.72 \pm 4.73 \%$ in the combined group. Compared with the remaining three groups, the apoptotic rate of the combined group was significantly increased $(\mathrm{P}<0.05)$.

Effect of icotinib on cell cycle following radiation. The influence of icotinib in combination with or without radiotherapy on the HT29 and HCT116 cell cycle was detected via flow cytometry (Fig. 4). In HT29 cells, the G2/M phase of the single drug group was $10.45 \pm 0.89 \%$, while it was $21.50 \pm 1.99 \%$ in the RT alone group and $35.79 \pm 1.10 \%$ in the combined group. The percentage of cells in the G2/M phase in the combined group was higher than that of the single drug or RT alone group $(\mathrm{P}<0.05)$. For HCT116 cells, the $\mathrm{G} 2 / \mathrm{M}$ phase of the single drug group was $12.77 \pm 2.03 \%$, while that of the RT alone group was $68.53 \pm 2.49 \%$ and that of the combined group was $74.00 \pm 1.33 \%$. The combined group showed significantly longer arrest in the G2/M phase compared with the single drug group and a tendency to stagnate in the G2/M phase compared with the RT group, although without significant differences ( $\mathrm{P}>0.05$ ). Icotinib combined with RT treatment re-adjusted the cell cycle distribution, significantly increased sensitive cells and improved the effect of radiotherapy.

Effect of icotinib on $\gamma-H 2 A X$ and 53BP1 foci formation following radiotherapy. The present results showed that, 

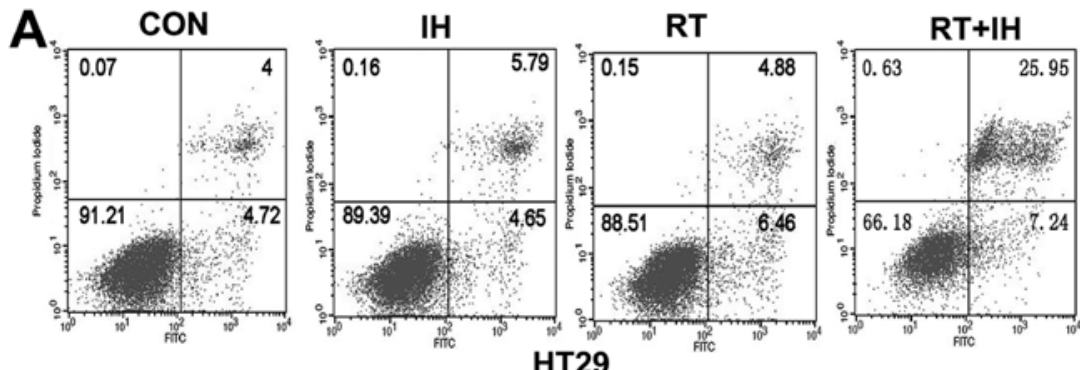

HT29
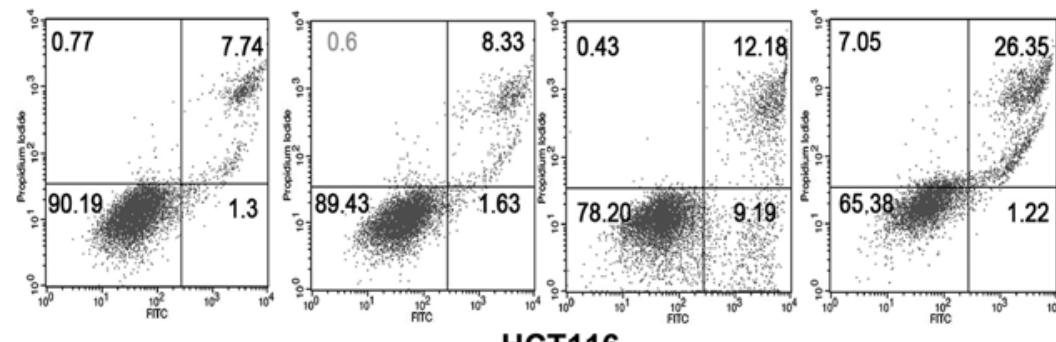

B

Treatment

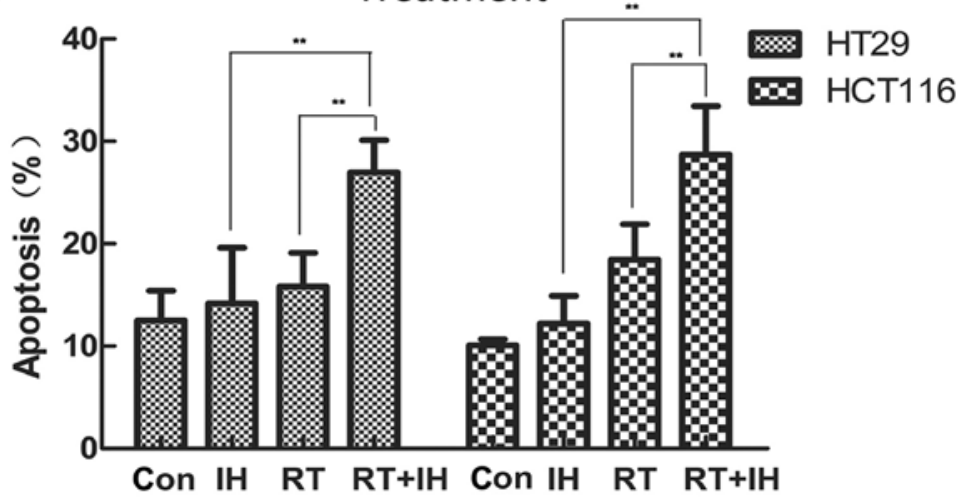

Figure 3. Apoptosis distribution of HT29 and HCT116 cells following different treatments. (A) Apoptosis was determined by flow cytometry in HT29 and HCT116 cells. (B) The apoptotic rate was calculated as the percentage of Annexin V-FITC-positive cells. Columns, mean; Error bars, SEM, from three independent experiments. ${ }^{* *} \mathrm{P}<0.01$; Con, control group; $\mathrm{IH}$, icotinib alone; $\mathrm{RT}$, radiotherapy alone; $\mathrm{RT}+\mathrm{IH}$, combined group.

$\mathrm{H} 2 \mathrm{AX}$ was rapidly phosphorylated in the presence of DSB and aggregated at the DSB, making it an important symbol of DNA DSB, as well as an important method to detect DNA DSB repairability. Twenty-four hours after the various treatments, the $\gamma$-H2AX foci per cell were detected (Fig. 5). The results showed that the combination groups showed a significantly higher number of unrepaired double-stranded DNA in the HT29 and HCT116 cell lines, while the RT alone or combined treatment with icotinib group showed significant differences as compared to the combined group $(\mathrm{P}<0.01)$. In the drug-RT combination group, the number of $\gamma$-H2AX foci was significantly higher than that in the RT alone group.

53BP1 has been proven to participate in double-stranded DNA repair, and is an important early regulator of DNA damage. To further determine whether 53BP1 is involved in double-stranded DNA repair during tumour suppression, the present study detected 53BP1 expression in HCT116 and HT29 cells treated in different groups (Fig. 6). Similar to $\gamma$-H2AX expression in different treatment groups, 53BP1 exhibited the same trends in the two differently treated cell lines. Tumour cells in the icotinib-RT combined group showed high levels of 53BP1 foci/cell that significantly differed from the RT, single drug and control groups. 53BP1 expression was significantly increased in the icotinib-RT combined group compared to the RT alone group and drug treatment group $(\mathrm{P}<0.01)$. The results are in concordance with the $\gamma-\mathrm{H} 2 \mathrm{AX}$ data presented for each group, suggesting that 53BP1 and $\gamma-\mathrm{H} 2 \mathrm{AX}$ are involved in DNA double-strand repair and are involved in the tumour response to treatment and radiosensitisation.

Icotinib hydrochloride increases the expression of 53BPI and $\gamma-H 2 A X$ when combined with radiotherapy. To determine the factors that contribute to impaired DSB repair, the responses of the 53BP1 and $\gamma$-H2AX proteins, which play key roles in DSB repair, were determined. Western blots were used to detect the expression of $\gamma-\mathrm{H} 2 \mathrm{AX}$ and 53BP1 protein in human colorectal cancer cells following interference in the different groups. As shown in Fig. 7, icotinib-RT combined treatment significantly increased the expression levels of $\gamma-\mathrm{H} 2 \mathrm{AX}$ and 53BP1 proteins, suggesting that the combined treatment increased the DNA double-strand breaks, attenuated DNA repair and improved the effect of radiotherapy.

Antitumour activity of icotinib combined with radiotherapy on human colorectal cancer subcutaneous tumour xenograft. IH was shown to significantly inhibit HT29 and HCT116 cell 


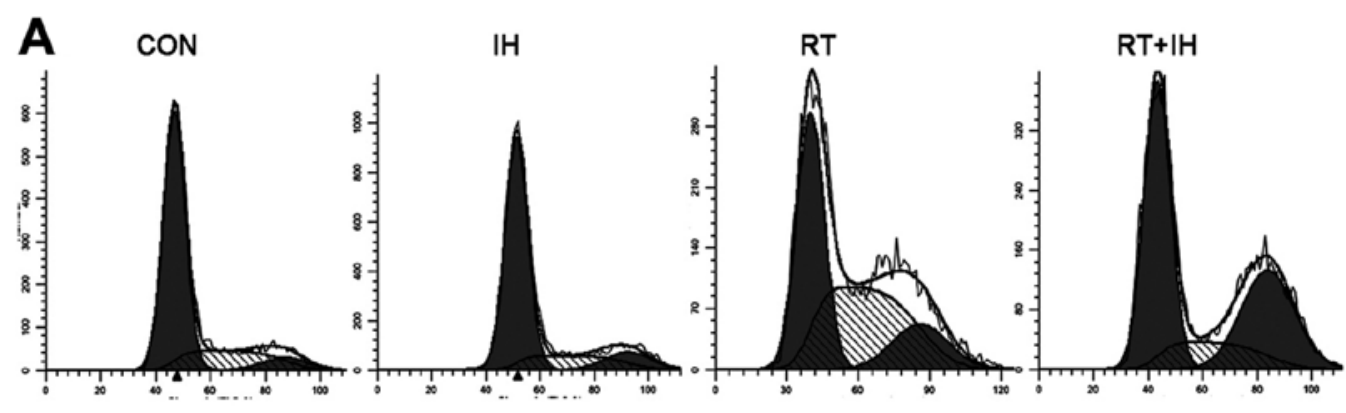

HT29

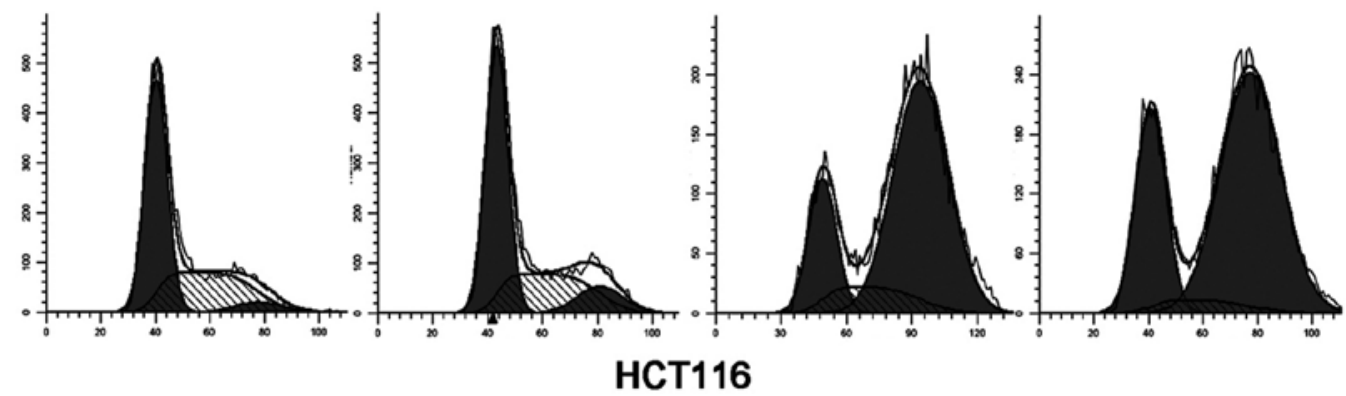

B

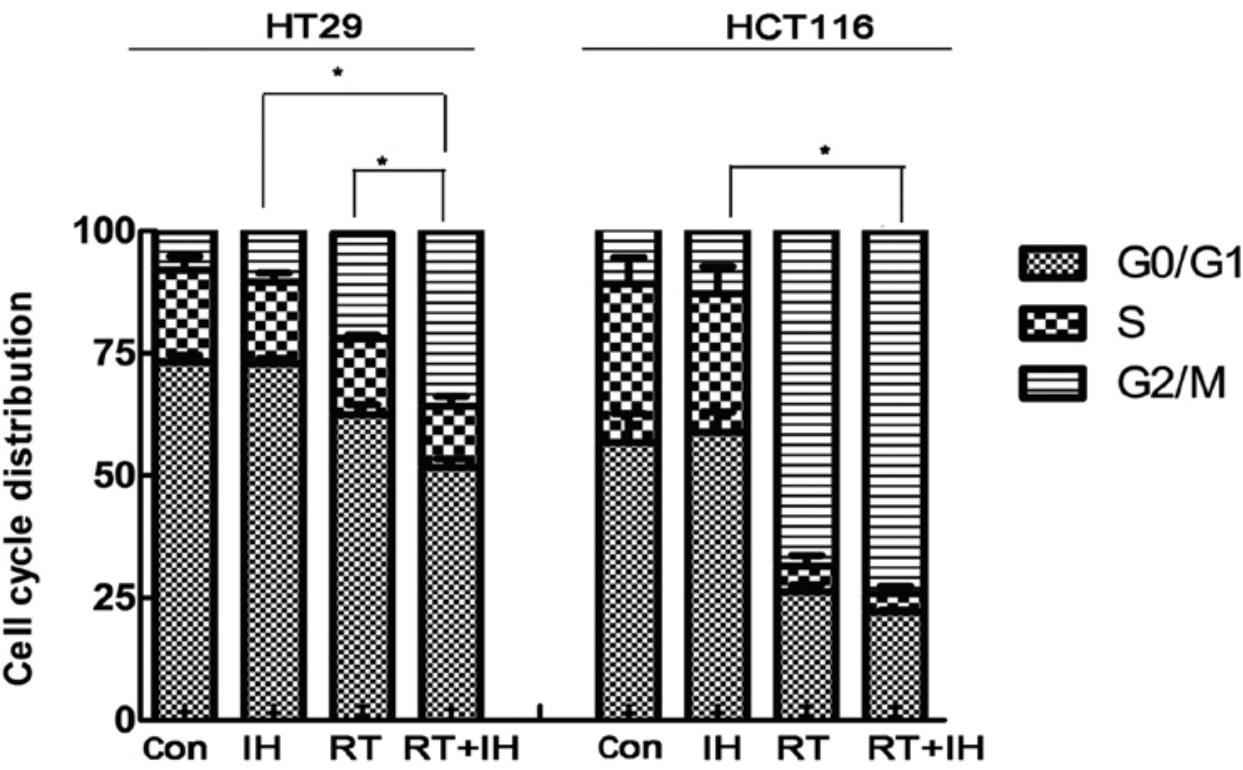

Figure 4. Cell cycle distribution was determined by flow assays in HT29 and HCT116 cells following different treatments. (A) Cells arrested in G2/M. (B) Histogram plots of cell cycle distribution, Columns, mean; Error bars, SEM, from three independent experiments. "P<0.05; Con, control group; IH, icotinib alone; RT, radiotherapy alone; $\mathrm{RT}+\mathrm{IH}$, combined group.

xenograft growth (Fig. 8). The final tumour volume of the combination group was statistically significantly smaller than that of the other groups $(\mathrm{P}<0.05)$ in both the HCT116 and HT29 models. This finding indicates that icotinib in combination with radiotherapy significantly inhibited the growth of colorectal cancer tumours.

\section{Discussion}

EGFR is a tyrosine kinase receptor of the ErbB family, and the signalling pathways in which it is involved regulate many important cell functions, including cell proliferation and apoptosis (22). Inhibition of the EGFR signalling pathway can reportedly improve the radiation effects $(23,24)$, but inhibition of the EGFR signalling pathway for the enhancement of the efficacy and mechanisms of colorectal cancer radiotherapy requires additional study. $\mathrm{IH}$ is a new oral epidermal growth factor receptor tyrosine kinase inhibitor, a quinazoline-type drug, with targets and mechanism similar to those of gefitinib. It is a reversible EGFR intracellular tyrosine tyrosine kinase inhibitor and has shown efficacy equal to that of gefitinib $(17,18)$. In vitro studies have already demonstrated a significant inhibitory effect on colon cancer cells. However, its superiority or equivalence to the effect of gefitinib when combined with radiation therapy remains unclear.

Since the abnormal expression of certain genes (such as $K-R A S)$ in the EGFR signalling pathway affects the efficacy of EGFR inhibitors $(13,14)$, the present study was conducted 


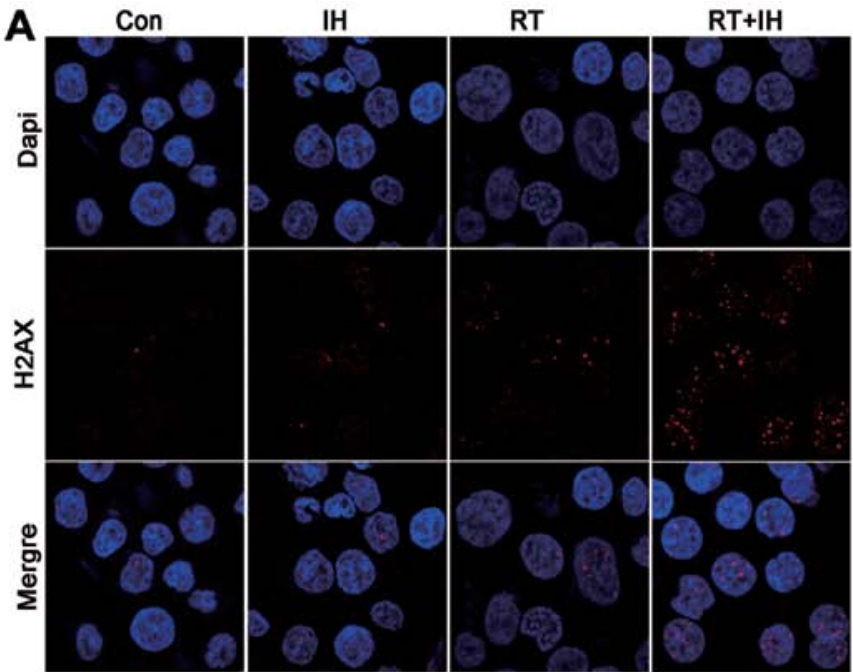

HT29

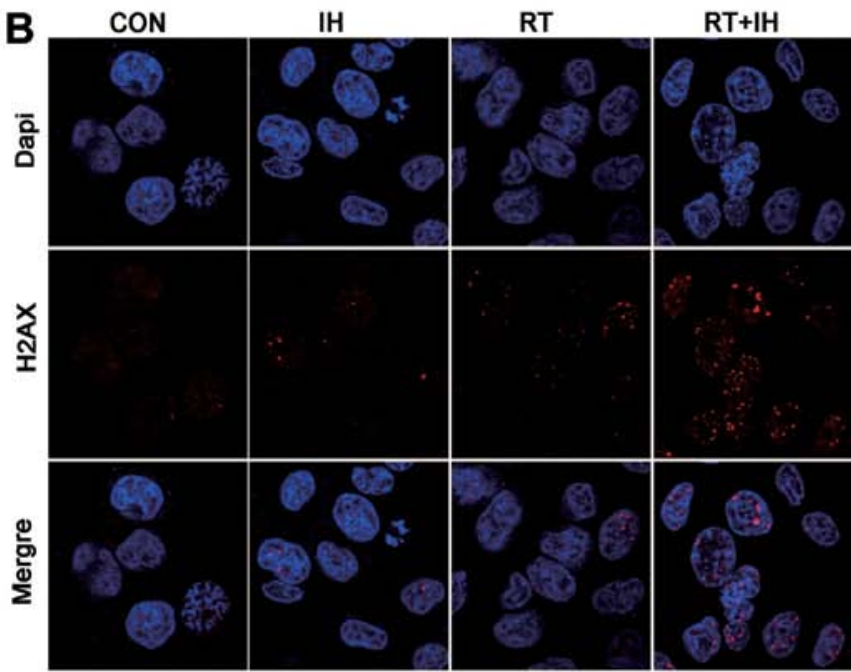

HCT116

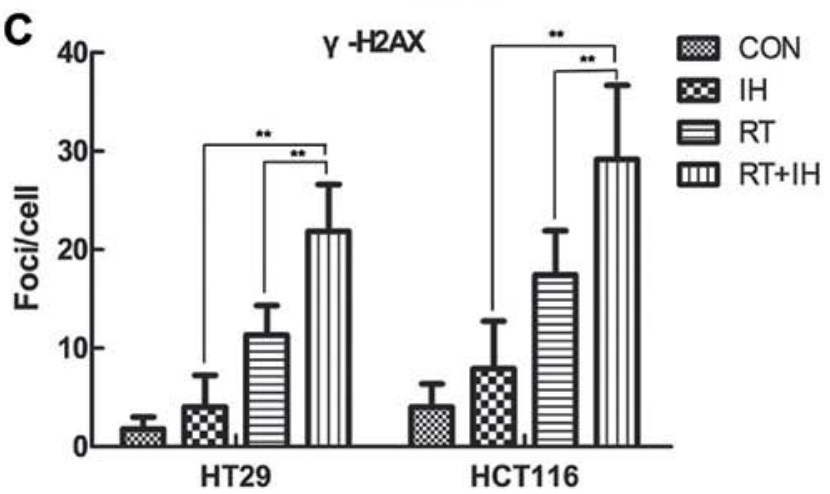

Figure 5. $\gamma-\mathrm{H} 2 \mathrm{AX}$ foci expression was detected in HT29 and HCT116 cells by immunofluorescent staining. (A) Immunofluorescent staining of $\gamma$-H2AX in HT29 cells (x1,000). (B) Immunofluorescent staining of $\gamma$-H2AX in HCT116 cells $(x 1,000)$. (C) Quantitative detection of $\gamma-\mathrm{H} 2 \mathrm{AX}$ foci number, Columns, means; Error bars, SEM, from three independent experiments. * $\mathrm{P}<0.01$; Con, control group; IH, icotinib alone; RT, radiotherapy alone; $\mathrm{RT}+\mathrm{IH}$, combined group.

on the $K-R A S$ gene expression status. K-RAS wild-type HT-29 cell line and the K-RAS mutant HCT-116 cell line were selected to establish wild- and mutant-type colorectal cancer in vitro and in vivo models. At the cell level, the impact of icotinib on the radiosensitivity of these two colorectal cancer

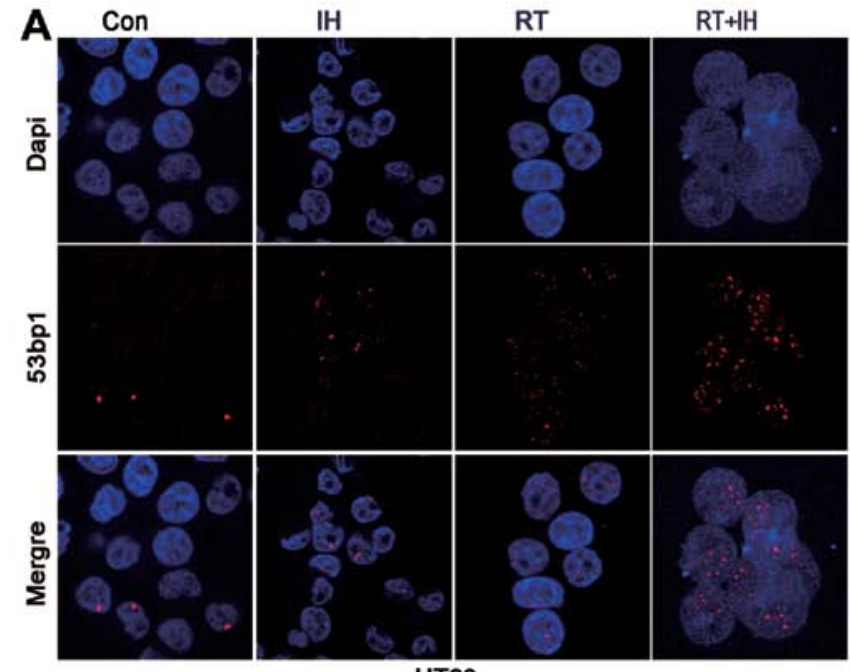

HT29
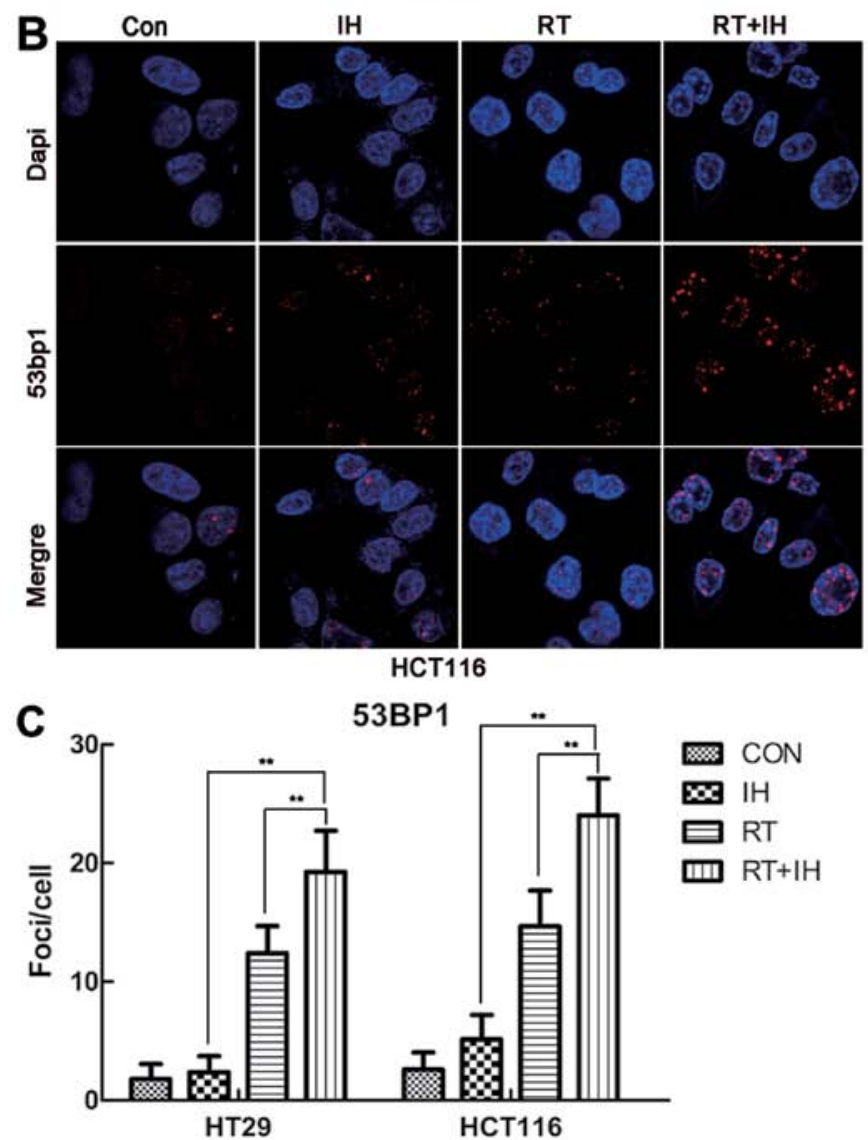

Figure 6. 53BP1 foci expression was detected in HT29 and HCT116 cells by immunofluorescence staining. (A) Immunofluorescent staining of 53BP1 in HT29 cells (x1,000). (B) Immunofluorescent staining of 53BP1 in HCT116 cells $(\mathrm{x} 1,000)$. (C) Quantitative detection of 53BP1 foci number, Columns, means; Error bars, SEM, from three independent experiments. ${ }^{* *} \mathrm{P}<0.01$; Con, control group; IH, icotinib alone; RT, radiotherapy alone; RT+IH, combined group.

lines was studied via a clone formation experiment in the present study. Moreover, icotinib with gefitinib was compared to understand sensitising differences between the two TKI inhibitors in colorectal cancer. Furthermore, tumour-bearing mouse models were established to understand the effect of icotinib combined radiotherapy treatment on tumour proliferation. The results showed that icotinib and gefitinib promoted 

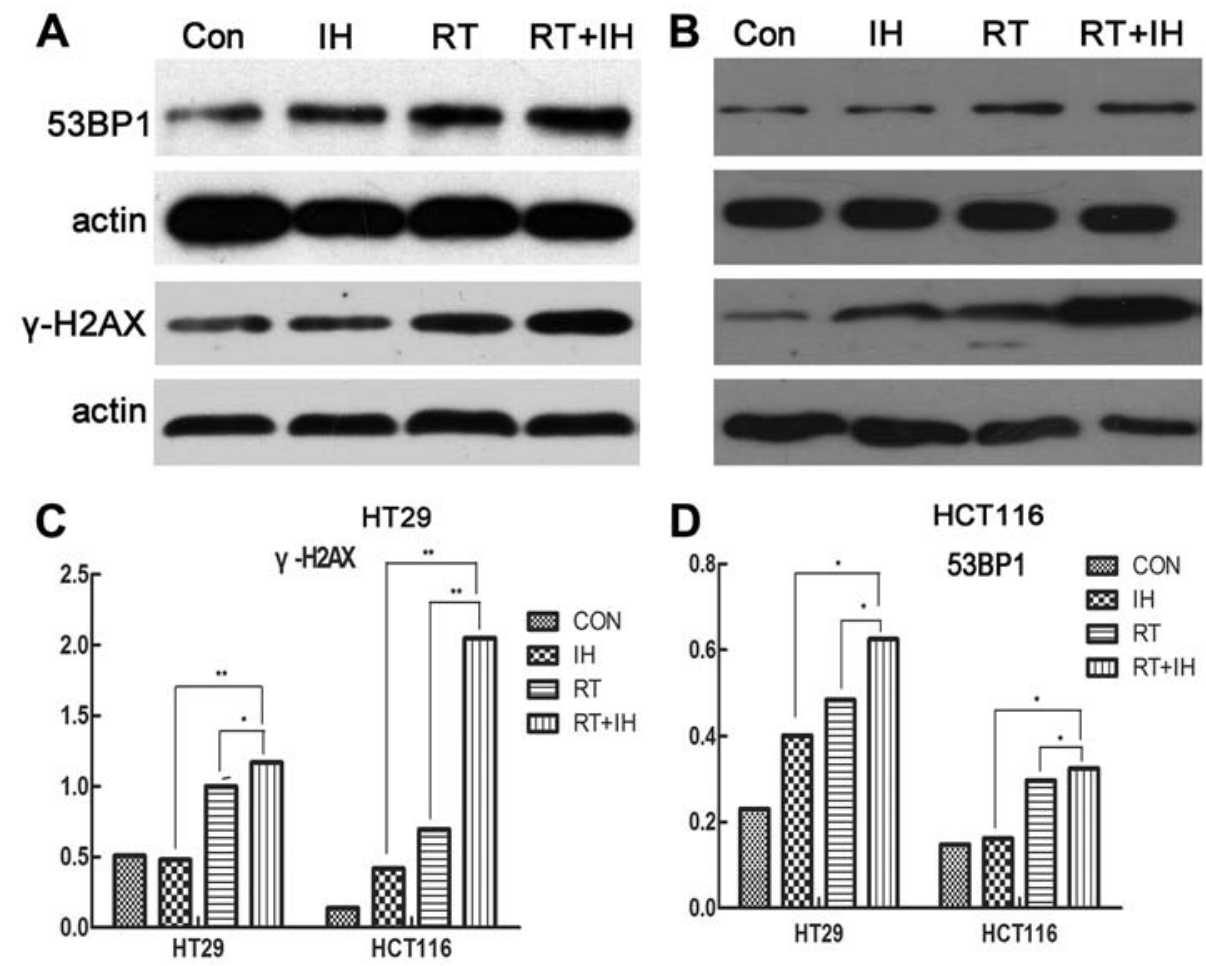

Figure 7. Effect of icotinib on the expression of $\gamma-\mathrm{H} 2 \mathrm{AX}$ and 53BP1 was detected by western blotting. (A) Expression levels of $\gamma-\mathrm{H} 2 \mathrm{AX}$ and 53BP1 proteins in HT29 cells were detected by western blotting for the four groups of treatment. (B) Expression of $\gamma-\mathrm{H} 2 \mathrm{AX}$ and 53BP1 protein in HCT116 cells detected by western blotting for the four groups of treatment. (C and D) Quantitative detection of $\gamma$-H2AX and 53BP1 bar chart. Columns, means; Error bars, SEM, from three independent experiments. ${ }^{*} \mathrm{P}<0.05,{ }^{* *} \mathrm{P}<0.01$; Con, control group; IH, icotinib alone; RT, radiotherapy alone; RT+IH, combined group.
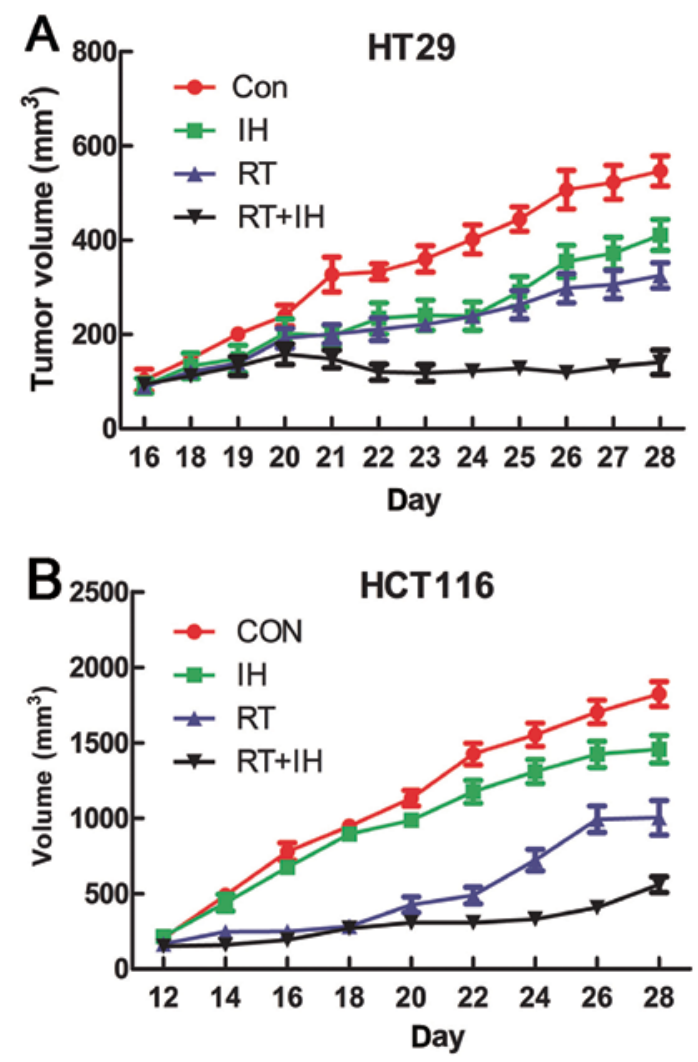

Figure 8. Inhibition of in vivo tumour growth in nude mice treated with radiotherapy alone (RT: $5 \times 2$ Gy, days 1-5), icotinib alone and their combination (IH+RT) compared with control without treatment. Tumour growth of (A) HT-29 and (B) HCT-116 cells following different treatments. Data points are the mean values obtained from 6-8 animals. Error bars are the SEM of six experiments. the radiosensitivity of the two colon cancer cells in vitro compared to radiotherapy alone, and their sensitising effect was not influenced by $K-R A S$ gene expression. Compared with gefitinib, icotinib significantly increased the radiosensitivity of tumour cells, confirming the superiority of icotinib to gefitinib. In vivo, icotinib was shown to produce significant inhibition of HT29 and HCT116 cell xenograft growth. In addition, the inhibitory effect in the combination treatment with icotinib and radiotherapy group was better than that of icotinib alone group and better than the radiotherapy alone group. Thus, icotinib combined with radiotherapy induced a highly significant inhibition of tumour growth and realized the radiosensitisation effectively to colorectal cancer in the present study.

Generally, radiosensitivity is governed by the capacity of the cell for efficient repair of radiation-induced lesions in the DNA, mainly the repair of DSBs. DNA double-strand breaks are a lethal cell injury, and radiation can induce DSB formation in tumour cells, interfering with DSB repair. Thus, increasing the extent of DSB damage is a radiosensitisation strategy $(21,25)$. In the presence of DSB, histone H2AX rapidly phosphorylates tryptophan 139 and aggregates at the DSB, forming visible fluorescence foci (foci) (26). Other components of DNA damage, including phosphorylated ATM, phosphorylated DNA-PKcs, 53BP1, BRCA1, MDC1, RAD51 and MRE11/RAD50/NBS1 (MRN complex), all subsequently participate in the repair (25). Previous studies have shown that the initial number of foci is associated with the theoretical number of radiation-induced foci, and $\gamma$-H2AX will subsequently be dephosphorylated and abolished with the elimination of DNA damage (such as being repaired) (27). 
Therefore, fluorescently labelled $\gamma-\mathrm{H} 2 \mathrm{AX}$ was considered to be an effective tool to distinguish intracellular DSB, where the intracellular amount of residual $\gamma-\mathrm{H} 2 \mathrm{AX}$ foci, which represents the cell damage repair capacity, depended on the cell type and is closely associated with cell radio-sensitivity (28).

In our study, tumour cell apoptosis in different treatment groups was detected by flow cytometry. The results showed that icotinib increased the apoptotic rate of HCT29 and HT116 cells following radiotherapy treatment compared to the drug and radiotherapy alone groups. Furthermore, the residual $\gamma$-H2AX foci in different treatment groups were examined in HCT29 and HT116 cells using immunofluorescent staining. The results showed that icotinib combined with radiotherapy significantly increased the intracellular $\gamma$-H2AX foci compared with the drug group and radiotherapy alone group. Western blot analysis indicated that icotinib significantly increased the intracellular expression of $\gamma-\mathrm{H} 2 \mathrm{AX}$ protein after radiotherapy treatment, which suggests that icotinib in combination with radiotherapy increases the intracellular DSB, thereby inducing tumour cell apoptosis.

Studies have shown that EGFR-TKI can affect the key components of the DNA repair pathway to increase DNA damage in order to achieve a radiosensitisation effect (21). 53BP1 has been proven to play an important intermediary role in DSB repair, while it is important in the cell response to treatment $(29,30)$. The 53BP1 protein consists of two Tudor structural domains and a C-end BRCT domain, with the former domain allowing it to aggregate at DSB, while the latter domain is involved in the interactive response with other DNA damage repair proteins $(31,32)$. DNA DSBs activate ataxia mutations (ataxia telangiectasia-mutated, ATM) of capillaries, induce a strong phosphorylation of 53BP1, and aggregate at DSB via the Tudor end and phosphorylated histone H2AX $(\gamma-\mathrm{H} 2 \mathrm{AX})$ and other proteins associated with repair damage. The C-terminal end activates cell cycle checkpoint kinase 1 (checkpoint kinase-1, CHK1) and cell cycle checkpoint kinase 2 (checkpoint kinase-2, CHK2) to regulate the cell cycle G1/S, S and G2/M checkpoints $(32,33)$. ATM-CHK2 activation further phosphorylated P53 and induced apoptosis, which depends on P53. Studies have reported that different levels of 53BP1 expression can interfere with cell cycle distribution and influence the treatment response. For example, Li et al found that a high expression of 53BP1 can induce the breast cancer cell cycle to stagnate in the $\mathrm{G} 2 / \mathrm{M}$ phase, thus enhancing the sensitivity of tumour cells to subsequent drug treatment. However, cells with a low expression of 53BP1 exhibited resistance to treatment (34). EGFR inhibitors can reportedly cause tumour cell cycle redistribution and increase the percentage of G2/M- or G1-phase cells while decreasing the proportion of S-phase cells (35). Therefore, EGFR inhibitors may influence the expression of 53BP1 and radiosensitise cells by interfering with the cell cycle distribution.

Using flow cytometry to detect cell cycle distribution, we found that icotinib in combination with radiotherapy adjusts the cell cycle distribution of wild-type HT29 colon cancer cells and reduces the proportion of cells in the G0/G1 phase in order to maximise the number of cells in G2/M arrest and facilitate the apoptosis or necrosis of radiosensitive cells For HCT116 colon cancer cells, radiotherapy treatment or icotinib combined with radiotherapy treatment decreased the proportions of cells in the G0/G1 and S phases and significantly increased G2/M-phase arrest. Although the two treatments did not produce statistically significant differences, an apparent increasing trend for G2/M-phase arrest was observed in the combined treatment group.

After further testing the 53BP1 foci of HCT29 and HT116 cells following different interventions in the treatment using immunofluorescent staining, the results showed that icotinib in combination with radiotherapy significantly increased intracellularly by the 53BP1 foci compared with the drug group and radiotherapy alone group. Western blot analysis revealed that icotinib significantly increased the intracellular 53BP1 expression after radiotherapy, suggesting that icotinib may alter the cell cycle by increasing the intracellular 53BP1 expression, and radiosensitise cells by inducing apoptosis.

In conclusion, we have shown that $\mathrm{IH}$, a potent inhibitor of EGFR tyrosine kinase activity, at pharmacologically achievable levels, may sensitise tumour cells to radiation by influencing key DSB repair proteins and delaying DNA damage repair. This finding suggests an interaction between EGFR signaling pathways and the regulation of DSBs repair in the nucleus. Examination of these interactions may reveal additional strategies for radiosensitising human tumour cells or biomarkers for identifying patients who may benefit from the combination of molecularly targeted agents and radiotherapy.

\section{References}

1. Secco GB, Fardelli R, Rovida S, et al: Is intensive follow-up really able to improve prognosis of patients with local recurrence after curative surgery for rectal cancer? Ann Surg Oncol 7: 32-37, 2000

2. Salo JC, Paty PB, Guillem J, Minsky BD, Harrison LB and Cohen AM: Surgical salvage of recurrent rectal carcinoma after curative resection: a 10-year experience. Ann Surg Oncol 6: 171-177, 1999.

3. Pilipshen SJ, Heilweil M, Quan SH, Sternberg SS and Enker WE: Patterns of pelvic recurrence following definitive resections of rectal cancer. Cancer 53: 1354-1362, 1984.

4. Shin SJ, Yoon HI, Kim NK, et al: Upfront systemic chemotherapy and preoperative short-course radiotherapy with delayed surgery for locally advanced rectal cancer with distant metastases. Radiat Oncol 6: 99, 2011.

5. Bosset JF, Collette L, Calais G, et al: Chemotherapy with preoperative radiotherapy in rectal cancer. N Engl J Med 355: 1114-1123, 2006

6. Rodel C, Liersch T, Becker H, et al: Preoperative chemoradiotherapy and postoperative chemotherapy with fluorouracil and oxaliplatin versus fluorouracil alone in locally advanced rectal cancer: initial results of the German CAO/ARO/AIO-04 randomised phase 3 trial. Lancet Oncol 13: 679-687, 2012.

7. Baumann $M$ and Krause M: Targeting the epidermal growth factor receptor in radiotherapy: radiobiological mechanisms, preclinical and clinical results. Radiother Oncol 72: 257-266, 2004.

8. Ullrich A and Schlessinger J: Signal transduction by receptors with tyrosine kinase activity. Cell 61: 203-212, 1990.

9. Galizia G, Lieto E, Ferraraccio F, et al: Prognostic significance of epidermal growth factor receptor expression in colon cancer patients undergoing curative surgery. Ann Surg Oncol 13: 823-835, 2006

10. Porębska I, Harłozińska A and Bojarowski T: Expression of the tyrosine kinase activity growth factor receptors (EGFR, ERB B2, ERB B3) in colorectal adenocarcinomas and adenomas. Tumour Biol 21: 105-115, 2000.

11. Milas L, Mason K, Hunter N, et al: In vivo enhancement of tumor radioresponse by $\mathrm{C} 225$ antiepidermal growth factor receptor antibody. Clin Cancer Res 6: 701-708, 2000.

12. Harari PM and Huang SM: Head and neck cancer as a clinical model for molecular targeting of therapy: combining EGFR blockade with radiation. Int J Radiat Oncol Biol Phys 49: 427-433, 2001. 
13. Dewdney A, Cunningham D, Tabernero J, et al: Multicenter randomized phase II clinical trial comparing neoadjuvant oxaliplatin, capecitabine, and preoperative radiotherapy with or without cetuximab followed by total mesorectal excision in patients with high-risk rectal cancer (EXPERT-C). J Clin Oncol 30: $1620-1627,2012$.

14. Shin HK, Kim M-S, Lee JK, et al: Combination effect of cetuximab with radiation in colorectal cancer cells. Tumori 96 $713,2010$.

15. Williams KJ, Telfer BA, Stratford IJ and Wedge SR: ZD1839 ('Iressa'), a specific oral epidermal growth factor receptortyrosine kinase inhibitor, potentiates radiotherapy in a human colorectal cancer xenograft model. Br J Cancer 86: 1157-1161, 2002.

16. Häggblad Sahlberg S1, Spiegelberg D, Lennartsson J, Nygren P, Glimelius B and Stenerlöw B: The effect of a dimeric Affibody molecule $\left(Z_{\mathrm{EGFR}: 1907) 2}\right.$ targeting EGFR in combination with radiation in colon cancer cell lines. Int J Oncol 40: 176-184, 2012

17. Tan F, Shen X, Wang D, et al: Icotinib (BPI-2009H), a novel EGFR tyrosine kinase inhibitor, displays potent efficacy in preclinical studies. Lung Cancer 76: 177-182, 2012.

18. Shi Y, Zhang L, Liu X, et al: Icotinib versus gefitinib in previously treated advanced non-small-cell lung cancer (ICOGEN): a randomised, double-blind phase 3 non-inferiority trial. The Lancet Oncol 14: 953-961, 2013.

19. Santivasi WL and Xia F: Ionizing radiation-induced DNA damage, response, and repair. Antioxid Redox Signal 21: 251-259, 2014.

20. Qu YY, Hu SL, Xu XY, et al: Nimotuzumab enhances the radiosensitivity of cancer cells in vitro by inhibiting radiation-induced DNA damage repair. PLoS One 8: e70727, 2013.

21. Tanaka T, Munshi A, Brooks C, Liu J, Hobbs ML and Meyn RE: Gefitinib radiosensitizes non-small cell lung cancer cells by suppressing cellular DNA repair capacity. Clin Cancer Res 14: 1266-1273, 2008.

22. Herbst RS and Bunn PA Jr: Targeting the epidermal growth factor receptor in non-small cell lung cancer. Clin Cancer Res 9 5813-5824, 2003.

23. Dittmann K, Mayer C, Fehrenbacher B, et al: Radiation-induced epidermal growth factor receptor nuclear import is linked to activation of DNA-dependent protein kinase. J Biol Chem 280: 31182-31189, 2005.
24. Sano D, Kawakami M, Fujita K, et al: Antitumor effects of ZD6474 on head and neck squamous cell carcinoma. Oncol Rep 17: 289-295, 2007.

25. Rothkamm K and Löbrich M: Evidence for a lack of DNA double-strand break repair in human cells exposed to very low x-ray doses. Proc Natl Acad Sci USA 100: 5057-5062, 2003.

26. Burma S, Chen BP, Murphy M, Kurimasa A and Chen DJ: ATM phosphorylates histone $\mathrm{H} 2 \mathrm{AX}$ in response to DNA double-strand breaks. J Biol Chem 276: 42462-42467, 2001.

27. Mah LJ, Orlowski C, Ververis K, Vasireddy RS, El-Osta A and Karagiannis TC: Evaluation of the efficacy of radiationmodifying compounds using $\gamma \mathrm{H} 2 \mathrm{AX}$ as a molecular marker of DNA double-strand breaks. Genome Integr 2: 3, 2011.

28. Lobrich M, Shibata A, Beucher A, et al: gammaH2AX foci analysis for monitoring DNA double-strand break repair: strengths, limitations and optimization. Cell Cycle 9: 662-669, 2010.

29. Ward IM, Difilippantonio S, Minn K, et al: 53BP1 cooperates with $\mathrm{p} 53$ and functions as a haploinsufficient tumor suppressor in mice. Mol Cell Biol 25: 10079-10086, 2005.

30. Bouwman P, Aly A, Escandell JM, et al: 53BP1 loss rescues BRCA1 deficiency and is associated with triple-negative and BRCA-mutated breast cancers. Nat Struct Mol Biol 17: 688-695, 2010.

31. Nuciforo PG, Luise C, Capra M, Pelosi G and d'Adda di Fagagna F: Complex engagement of DNA damage response pathways in human cancer and in lung tumor progression. Carcinogenesis 28 : 2082-2088, 2007.

32. Clarke A, Jones N, Pryde F, Adachi Y and Sansom O: 53BP1 deficiency in intestinal enterocytes does not alter the immediate response to ionizing radiation, but leads to increased nuclear area consistent with polyploidy. Oncogene 26: 6349-6355, 2007.

33. Morales JC, Franco S, Murphy MM, et al: 53BP1 and p53 synergize to suppress genomic instability and lymphomagenesis. Proc Natl Acad Sci USA 103: 3310-3315, 2006.

34. Li X, Xu B, Moran MS, et al: 53BP1 functions as a tumor suppressor in breast cancer via the inhibition of NF- $\kappa B$ through miR-146a. Carcinogenesis 33: 2593-2600, 2012.

35. Park JS, Jun HJ, Cho MJ, et al: Radiosensitivity enhancement by combined treatment of celecoxib and gefitinib on human lung cancer cells. Clin Cancer Res 12: 4989-4999, 2006. 